\title{
Vehicle odometry model identification considering dynamic load transfers
}

\author{
Máté Fazekas, Balázs Németh, Péter Gáspár and Olivier Sename
}

\begin{abstract}
The paper proposes a parameter identification method for a vehicle model using real measurements of onboard sensors. The motivation of the paper is to improve the localization of the vehicle when the accuracy of the regular methods is poor, e.g. in the case of unavailable GNSS signals, no enough feature for vision, or low acceleration for IMUbased techniques. In these situations the wheel encoder based odometry may be an appropriate choice for pose estimation, however, this method suffers from parameter uncertainty and unmodelled effects. The utilized vehicle model operates with dynamic wheel radius. The proposed identification method combines the Kalman-filter and least square techniques in an iterative loop for estimating the parameters. The estimation process is verified by real test of a compact car. The results are compared with the nominal setting, in which there is no estimation.
\end{abstract}

Index Terms-parameter identification, vehicle odometry, sensor fusion, localization, dynamic load transfer

\section{INTRODUCTION}

With the appearance of autonomous vehicle functions, vehicle localization become a key question in the automotive industry. The position and orientation estimation can be performed by several methods using a wide range of sensors, such as camera, LiDAR, GNSS, IMU and wheel encoders. The perception based methods demand prior teaching and well recognizable features, see [1]. The fusion of GNSS and IMU measurements could be precise at higher velocities, but it requires the current knowledge of the covariances of signals. Furthermore, the GNSS signal can be unavailable in some cases, e.g. parking in a garage, see [2], or among tower blocks in urban areas. In these situations vehicle model based odometry with wheel encoder measurements may be the appropriate choice for vehicle localization. However, this method suffers from parameter uncertainties, therefore the estimation of some vehicle parameters is a required capability.

In the automotive industry this type of localization appears with the parking assist functions [3]. The examination of

M. Fazekas, B. Németh and P. Gáspár are with Systems and Control Laboratory, Institute for Computer Science and Control, Kende u. 13-17, H-1111, Budapest, Hungary. E-mail: [mate.fazekas; balazs. nemeth; gaspar] @sztaki.hu

O. Sename is with GIPSA-lab, INPG, Université Grenoble Alpes, 11 Rue des Mathématiques, 38402 Grenoble, France. E-mail: olivier.senamelgipsa-lab.grenoble-inp. fr

The research was supported by the National Research, Development and Innovation Office through the project 'Integration of velocity and suspension control to enhance automated driving comfort in road vehicles' (NKFIH 2018-2.1.13-TÉT-FR)

The work of Máté Fazekas was partially supported by the ÚNKP-193 New National Excellence Program of the Ministry of Innovation and Technology. the used vehicle models is a well-explored area [3] and [4]. However, high accurate pose estimation requires wellcalibrated vehicle models, but the precise parameter identification remains a challenge. The parameter identification is an important task due to variations in the parameters resulted from wheel changing or tyre wear. A detailed examination can be found in our previous work in [5].

An examination of odometry errors is found in [6] and its correction method based on a set of well-defined and pre-programmed paths presented in [7] and [8]. Other algorithms use perception sensor measurements, e.g. vision, laser range finder or DGPS measurements, see [9]-[11]. The disadvantage of these methods is the application of expensive sensors. In a fully autonomous vehicle, it is desirable for self calibration to be done without any pre-programmed paths or specific marker. A self-adaptive identification method that uses only cost effective GNSS measurements and handles time delays of the sensors is proposed by [12].

The general method for state estimation is the well-known Kalman-filter (KF) and for parameter identification is the least squares (LS) method, see [13]. In a dynamic model both the state and parameter estimation can be handled in one filter, which is called Augmented Kalman-filter [9], [10], [12]. However the parameters values are modified in every timestep, therefore constant value is not achievable and the effect of noise is significant.

The scope of this paper is to identify the vehicle odometry parameters of a compact series car from real measurements considering dynamic load transfers. In Section II the twowheel model of the odometry is presented. The core of process is a unique solution, in which the Kalman-filter is responsible for state estimation and the parameters are identified with a non-linear least square technique in an iterative loop. The algorithm is demonstrated in detail in the Section IV. The advantages of the proposed method are the lower effect of the measurements noises and the estimation results in a stable value for the parameters. The test route is segmented into sub-traces and the stable identified values are obtained by computing its mean. The estimation results and tests can be found in the Section V. Finally the contributions of paper and future challenges are summarized in Section VI.

\section{ODOMETRY MODEL WITH DYNAMIC LOAD TRANSFER}

The dead-reckoning navigation is based on a model, of which state vector $\mathrm{x}_{k}$ contains the longitudinal and lateral vehicle positions of the reference point $x_{k}, y_{k}$, which can be the midpoint of rear track and the heading angle $\theta_{k}$. The 
position of the vehicle is calculated as

$$
\begin{aligned}
& x_{k}=x_{k-1}+v_{k} \cdot T \cdot \cos \left(\theta_{k-1}+\omega_{k} \cdot T / 2\right), \\
& y_{k}=y_{k-1}+v_{k} \cdot T \cdot \sin \left(\theta_{k-1}+\omega_{k} \cdot T / 2\right), \\
& \theta_{k}=\theta_{k-1}+\omega_{k} \cdot T .
\end{aligned}
$$

The inputs of system are the longitudinal $v_{k}$ and angular $\omega_{k}$ velocities, which are calculated from the rear wheels in the case of two-wheel odometry model as

$$
\begin{aligned}
v_{k} & =\left(n_{R L, k} \cdot c_{R L, k}+n_{R R, k} \cdot c_{R R, k}\right) /(2 \cdot T), \\
\omega_{k} & =\left(n_{R R, k} \cdot c_{R R, k}-n_{R L, k} \cdot c_{R L, k}\right) /\left(t_{R} \cdot T\right),
\end{aligned}
$$

where $n_{i}$ is the rotation number of the wheel, $c_{i, k}=2 \pi r_{i, k}$ is the actual wheel circumference, $t_{R}$ is the rear track and $T$ is the sampling time. $r_{i, k}$ is the rolling radius, define by dividing the longitudinal and angular speed assuming zero slip.

The slight change of the wheel radius due the effect of vertical dynamic is generally neglected, because the odometry based localization is widely used in low speed circumstances i.e. automated parking. However, the sensor measurements used for calibration are collected from normal city and suburb driving, where the dynamic is certainly higher. Therefore, the slight change due to the vertical load transfer should be considered, thus the current wheel circumferences used in our model are defined as

$$
\begin{aligned}
& c_{R L, k}=c_{e, R L}+D \cdot a_{y, k}, \\
& c_{R R, k}=c_{e, R R}-D \cdot a_{y, k},
\end{aligned}
$$

where the $c_{e, i}$ is the effective wheel circumference, $a_{y, k}$ is the lateral acceleration and $D$ is a parameter that takes into account the effect of vertical dynamics and will be descriebed as load transfer coefficient. Thus, the model is suitable to the parameter identification of a vehicle model, which is used in scenarios where input excitation is significant.

\section{Motivation of the dynamic wheel model}

The effect of this dynamic change is illustrated in the following example. The optimal parameter values are calculated by using a grid search method on a $476.8 \mathrm{~m}$ long sub-trace, which contains a left turn with $16 \mathrm{~m} / \mathrm{s}$ average speed, while the route and the lateral acceleration are illustrated in Figure 1. The model parameters are determined in two cases based
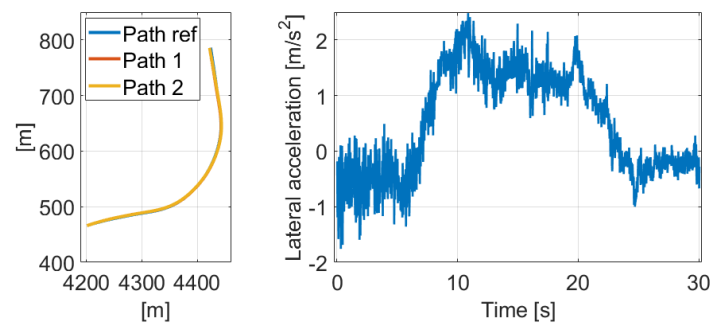

Fig. 1. Path and acceleration of the deviation effect example

on load transfer coefficients: first $D_{1}=0$ and second with adjustable parameter $D_{2}$. The optimal parameters and mean position errors are the following:

$$
\begin{array}{clll}
c_{e, R L 1}=1.96137 m & c_{e, R R 1}=1.96355 m & t_{R 1}=1.77 m \\
c_{e, R L 2}=1.96154 m & c_{e, R R 2}=1.96346 m & t_{R 2}=1.51 m \\
E r r_{1}=0.5510 m & \operatorname{Err}_{2}=0.5509 m & D_{2}=0.0011 \mathrm{~s}^{2}
\end{array}
$$

The optimal position errors are the same in each case and the $0.12 \%$ relative mean position error implies highly-accurate localization with this model integrating more than $450 \mathrm{~m}$. The effective circumferences are mainly the same, however, the optimal rear track values are very different, the real value is around $1.53 \mathrm{~m}$ from the datasheet of the vehicle. Therefore, the relatively low deviation parameter $D_{2}$ has a significant impact on the estimation, despite the fact that the circumference change is only $5 \mathrm{~mm}$ as it is shown in Figure 2. Thus, consideration of dynamic load transfers is important

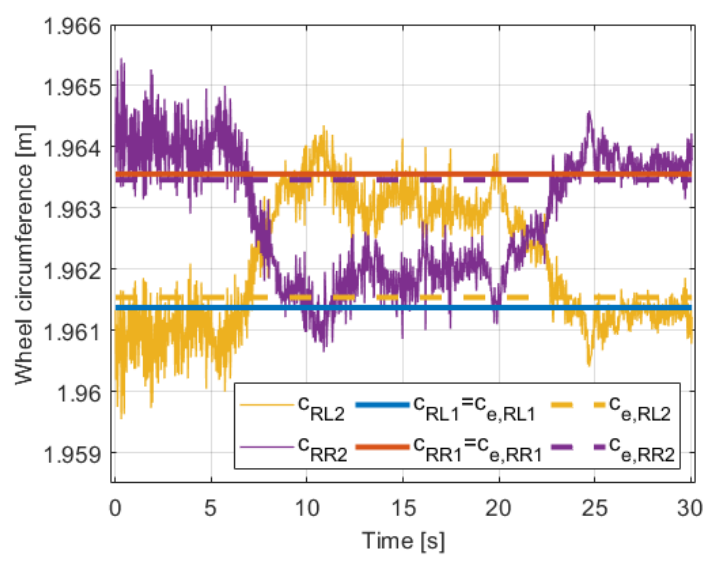

Fig. 2. Circumferences of the deviation effect example

in the two-wheel odometry model calibration.

\section{TEST VEHICLE AND MEASUREMENTS}

The test vehicle is an electric compact car which is equipped with GNSS, compass and IMU sensors and the wheel encoder signals from the vehicle CAN bus is also saved with a dSPACE MicroAutoBox and a Vector CAN Interface measurement setup. The sampling frequency is 25 $\mathrm{Hz}$, except the GNSS in which only $5 \mathrm{~Hz}$. The sensors are cost effective automotive grade types. The test track is a $11.82 \mathrm{~km}$ long way in suburban and city driving. The route contains several bends, two roundabouts and some crossroads. The longitudinal velocity is in the interval $0-20$ $\mathrm{m} / \mathrm{s}$. Because of the heavy traffic and lights it is highly varying with the average of $9.6 \mathrm{~m} / \mathrm{s}$. The yaw rate is between -0.7 and $0.7 \mathrm{rad} / \mathrm{s}$ and the lateral acceleration is between -4 and $4 \mathrm{~m} / \mathrm{s}^{2}$.

\section{A. Measurement fusion}

GNSS and IMU measurements are fused to reach absolute position and orientation values $\widetilde{\mathrm{x}}_{k}=\left[\widetilde{x}_{k}, \widetilde{y}_{k}, \widetilde{\theta}_{k}\right]^{T}$, which are utilized to identify the parameters. This fusion method has been investigated already in a wide range of papers 
considering the dynamic equation of $\ddot{p}=a$, where $p$ is the position and $a$ is the acceleration. The implemented method is similar to [14]. In this fusion the measurements of wheel encoders are not used.

\section{B. Route segmentation and selection}

The test track is divided into sub-traces for the identification to eliminate the effect of error accumulation. The procedure is based on time to provide equal number of measurement points for the LS estimation in each subtrace. The determination of subdivision length is based on two factors. If the value is low, the effect of actual pose measurement noises is high, which can result in a biased parameter identification. But, if the value is too high, the two wheel model is integrated over hundreds of meters, where the model can drift easily from the measurements, which results in incorrect estimation as well. However, the KF before the LS identification in the iterative loop eliminates the possibility of drift, thus the selected time length is 22.5 $\mathrm{s}$, which results in an average sub-trace length of $200 \mathrm{~m}$. Due to the fact that, the track and deviation parameters are contained just in the angular velocity equation, only the subtraces with higher than $0.15 \mathrm{rad} / \mathrm{s}$ absolute maximum yaw rate is selected for the identification.

\section{ITERATIVE PARAMETER IDENTIFICATION METHOD}

The accuracy of localization highly depends on the calibration of the vehicle model. The most significant parameters are the wheel circumferences, because the angular velocity is based on the difference of traveled distances by the wheels. Therefore, a little deviation from the true values may result in high error. The track width and load transfer coefficients are also important in the estimation in order to achieve a global optimum setting. The identification method operates with the Kalman-filtering for optimal state fusion and with the least square regression for estimating parameters.

The two-wheel odometry model in (1) results in the following nonlinear description

$$
\mathrm{x}_{k}=f\left(\mathrm{x}_{k-1}, \mathrm{u}_{k-1}, \vartheta\right),
$$

in which the inputs are the measured wheel rotation number $n_{i, k-1}$ of the encoders and the state vector contains the vehicle position and $\vartheta$ contains the parameters as

$$
\begin{aligned}
\mathrm{x}_{k} & =\left[\begin{array}{lll}
x_{k} & y_{k} & \theta_{k}
\end{array}\right]^{T}, \\
\mathrm{u}_{k} & =\left[\begin{array}{lll}
n_{R L, k} & n_{R R, k}
\end{array}\right], \\
\vartheta & =\left[\begin{array}{llll}
c_{e, R L} & c_{e, R R} & t_{R} & D
\end{array}\right] .
\end{aligned}
$$

The parameters are identified from position and orientation values, which are also estimated and indicated with

$$
\widetilde{\mathrm{x}}_{k}=\left[\begin{array}{lll}
\widetilde{x}_{k} & \widetilde{y}_{k} & \widetilde{\theta}_{k}
\end{array}\right]^{T} .
$$

The parameter identification method has two main layers, in which the Kalman-filtering and the least squares optimization are connected together in an iterative way. This approach can also be feasible for identification of Hammerstein and Wiener models, see in [15]. The process of iterative solution is illustrated in Figure 3.

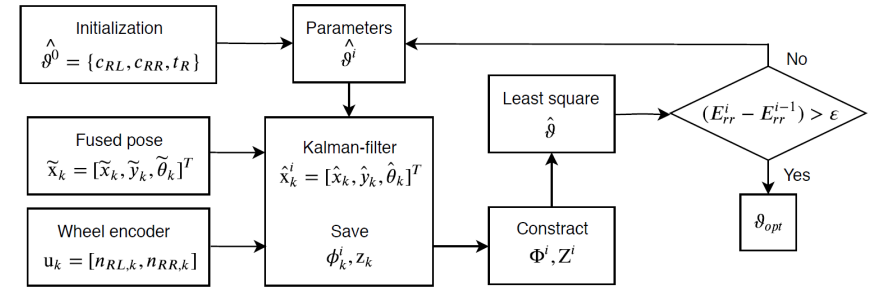

Fig. 3. Process of the iterative parameter identification (KF-LS identification)

\section{A. Kalman-filter for state estimation}

The core of method is an Extended Kalman-filter, which uses the wheel encoders as inputs and the fused position and orientation values as measurements. Moreover, the nonlinearity is approximated with the first derivative of the system model. The filtering of $\hat{\mathrm{x}}_{k}=\left[\hat{x}_{k}, \hat{y}_{k}, \hat{\theta}_{k}\right]^{T}$ is performed in two steps, where the prediction is based on the model $(8 \mathrm{~b})$, and in the innovation the measurements are utilized to improve the estimation (8e). The states and covariance matrix are calculated as

$$
\begin{aligned}
\hat{\mathrm{x}}_{k}^{-} & =f\left(\hat{\mathrm{x}}_{k-1}, \mathrm{u}_{k-1}\right) \\
\Sigma_{k}^{-} & =F_{k} \Sigma_{k-1} F_{k}^{T}+P, \\
G_{k} & =\Sigma_{k}^{-} H_{k}^{T}\left(H_{k} \Sigma_{k}^{-} H_{k}^{T}+M\right)^{-1}, \\
\hat{\mathrm{x}}_{k} & =\hat{\mathrm{x}}_{k}^{-}+G_{k}\left(\mathrm{y}_{k}-h\left(\hat{\mathrm{x}}_{k}^{-}\right)\right) \\
\Sigma_{k} & =\left(I-G_{k} H_{k}\right) \Sigma_{k}^{-},
\end{aligned}
$$

where the Jacobians are stated as follows and computed using the previous timestep values as

$$
F_{k}=\left.\frac{\partial f(\mathrm{x}, \mathrm{u})}{\partial \mathrm{x}}\right|_{\mathrm{x}=\hat{\mathrm{x}}_{k-1}, \mathrm{u}=\mathrm{u}_{k-1}}, \quad H_{k}=I,
$$

where $h(\mathrm{x})$ is the measurement equation, which is $h(\mathrm{x})=$ $\left[x_{k}, y_{k}, \theta_{k}\right]^{T}=\mathrm{y}_{k}=\widetilde{x}_{k} . G_{k}$ is the Kalman-gain factor, which ensures the optimal estimation of the states and guarantees the minimum covariance. The estimation highly depends on the $P$ and $M$ matrices, which are the model and measurements covariances.

\section{B. Least squares method for parameter estimation}

The parameters are identified with the least square regression method. The LS approximation is the conventional method when algebraic equations can be formed between the measurements and estimated parameters. The relationship is generally formulated as $\mathrm{y}_{k}=\phi_{k} \vartheta+\varepsilon_{k}$, where $\mathrm{y}_{k}$ is the measurement, $\phi_{k}$ contains algebraic functions and other measurements, $\varepsilon$ symbolizes the measurement noise and $\vartheta$ is the parameter vector to be identified. In the presented method the fused pose measurements $\widetilde{\mathrm{x}}_{k}$, the filtered states $\hat{\mathrm{x}}_{k}$ from the previous Kalman-filter and the vehicle model is used to formulate the LS equation as

$$
\underbrace{\left[\begin{array}{c}
\widetilde{x}_{k}-\hat{x}_{k-1} \\
\widetilde{y}_{k}-\hat{y}_{k-1} \\
\widetilde{\theta}_{k}-\hat{\theta}_{k-1}
\end{array}\right]}_{\mathrm{y}_{k}}=\underbrace{\left[\begin{array}{c}
v_{k} \cdot T \cdot \cos \left(\theta_{k-1}+\frac{\omega_{k} \cdot T}{2}\right) \\
v_{k} \cdot T \cdot \sin \left(\theta_{k-1}+\frac{\omega_{k} \cdot T}{2}\right) \\
\omega_{k} \cdot T
\end{array}\right]}_{g(\vartheta, k)} .
$$


The vehicle model results nonlinear problem, however linearization can be perform if initial $\vartheta_{0}$ value can be assumed. Using the Taylor-approximation as

$$
\mathrm{y}_{k}=g(\vartheta, k) \approx g\left(\vartheta_{0}, k\right)+\left.\frac{\partial g(\vartheta, k)}{\partial \vartheta}\right|_{\vartheta_{0}}\left(\vartheta-\vartheta_{0}\right),
$$

and introducing the following notations

$$
\begin{aligned}
& \mathrm{z}_{\mathrm{k}}=\mathrm{y}_{\mathrm{k}}-g\left(\vartheta_{0}, k\right)+\left.\frac{\partial g(\vartheta, k)}{\partial \vartheta}\right|_{\vartheta_{0}} \vartheta_{0}, \\
& \phi_{k}=\left.\frac{\partial g(\vartheta, k)}{\partial \vartheta}\right|_{\vartheta_{0}},
\end{aligned}
$$

the nonlinear estimation task with local linearization is transformed into the general linear LS form. From the $\mathrm{Z}_{\mathrm{k}}$ and $\phi_{k}$ values $\mathrm{Z}$ and $\Phi$ matrices are constructed, respectively. The estimated parameter vector is

$$
\hat{\vartheta}=\left(\Phi^{T} W \Phi\right)^{-1} \Phi^{T} W Z,
$$

where $W$ is a positive definite weighting matrix.

\section{Parameter identification in the iterative design}

New values are identified for the parameters with the LS method. However two facts must be taken into consideration. First, the Kalman-filtering operates with the actual parameters and the filtered states are used in the LS parameter identification, thus with the new parameters new LS problem is generated. Second, the used LS equation operate with the first order Taylor-coefficient of the nonlinear function, therefore the approximation is precise only close to the value used for local linearization. Thus, iterative method is proposed and the change of parameter values are limited in the iterations. The new value for the parameter vector is

$$
\hat{\vartheta}_{i}=\hat{\vartheta}_{i-1}+\alpha\left(\hat{\vartheta}-\hat{\vartheta}_{i-1}\right),
$$

where $i$ is the iteration step and $\alpha$ is a tuning parameter with the value of $0<\alpha \leq 1$ and it is named as learning rate. Position error with the resulted parameters is calculated from the fused measurements and the estimation is stopped, when the mean error starts to increase or the maximum iteration number is reached.

\section{Tuning method of the iterative process}

Generally the covariance matrices of the Kalman-filters $M$ and $P$ should be adjusted to the standard deviation of the measurements and used model. However, in practical applications these values are unknown, but the position is in meter and the angle is in radian, therefore the noise of angle is assumed to be lower. Furthermore, in our estimation the main goal is the identification of true parameter values and the KF must ensure this. During the iterations the model becomes more precise, therefore varying covariances are proposed as

$$
M=\operatorname{diag}([1,1,0.1]) \quad P=\operatorname{diag}\left(\left[\frac{p}{i^{q}}, \frac{p}{i^{q}}, \frac{0.01 p}{i^{q}}\right]\right)
$$

where $i$ is the actual iteration number and $q$ is a variable in the range of $1 \leq q \leq 2$, in this identification $q=1.3$ and $p=5$ is used.

In the LS estimation the change of parameter vector value must be limited between two iteration steps due to the linearization, thus $\alpha=0.1$ is chosen for learning rate. $W$ weighting matrix symbolizes the noise of equations and because only the ratio of values is significant the matrix is stated, such as $W=\operatorname{diag}\left(\left[1 / 1^{2}, 1 / 1^{2}, 1 / 0.1^{2}\right]\right)$, due to the effect of $1 \mathrm{~m}$ position and $1 \mathrm{rad}$ orientation error. Detailed explanations are in our previous work, see [5].

\section{E. Estimation sequence}

In Section II the illustrative example showed the interaction of the model parameters and the problem of the local optimum. Thus, the selection of appropriate initial parameters are necessary. Since there is no any apriori information about the tyre wear, equal values are assumed. However, the difference of circumferences has a high impact on the calculation of angular velocity. Therefore, first only the circumferences are identified and after the track and load transfer coefficient are estimated also.

\section{EXPERIMENTAL RESULTS}

In this section the proposed identification method is presented on the test track. The KF-LS based estimation is performed in each sub-trace. First the identification method is demonstrated on a sub-trace illustrating the iteration steps in details. Second, the method is presented on the case of circumference estimation and third, the estimation of the track and load transfer coefficient is illustrated. Some subtrace are eliminated, because the resulting position error or the estimated parameter values highly differ from the others. These may be the consequences of higher measurement noise on the initialization point of the sub-trace or the wheel slipping. The general indication of the parameter vector is $\vartheta_{s, i}^{t}$, where $s$ means the circumference estimation ( irc $)$ and the final identification ( $f i n)$ of every parameter, $i$ is the iteration number ( $i *$ is the optimal) and $t$ denotes the sub-trace number. The initial value for the parameters are the nominal ones, which are based on the wheel diameter and vehicle's datasheet. The values are $\left[c_{e, R L, \text { nom }}, c_{e, R R, \text { nom }}, t_{R, \text { nom }}, D_{\text {nom }}\right]=[2,2,1.55,0]$.

\section{A. Estimation method illustration}

The iterative identification procedure is presented in a given sub-trace in the case of circumference estimation. The mean position errors calculated from the fused measurements and the estimated circumferences can be found in Figure 4. At zero the error and circumferences indicates the nominal setting.

The nominal error is $21.13 \mathrm{~m}$, but with the identified values at iteration 10 the error is only $1.75 \mathrm{~m}$, thus the identification results 12 times better for the vehicle odometry localization. However, it is necessary to take into account that the resulted setting is related only to this sub-trace. Furthermore, in Figure 4 the slight difference between the 


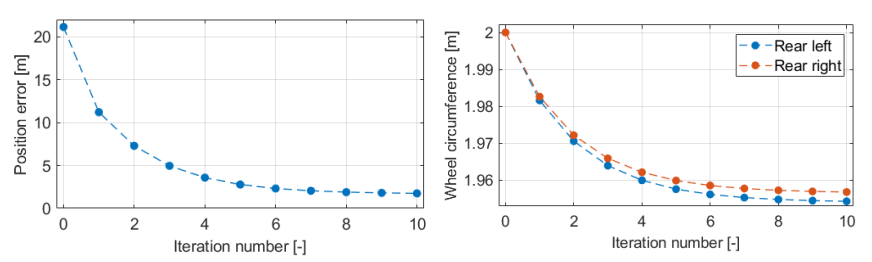

Fig. 4. Position error and estimated circumferences of the iterations

estimated circumferences can be seen, which have already appeared significantly at the third iteration. In Figure 5 the

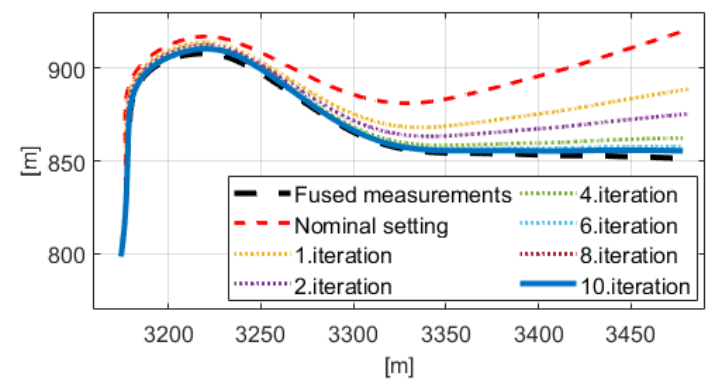

Fig. 5. Paths of the iterations

fused measurements, the nominal path and the paths of iterations can be found. The change is significant in the first few iteration and the resulted in path at the final iterations fits properly to the fused measurements.

\section{B. Initial wheel circumference estimation}

The same algorithm is used in every sub-trace for initial circumference estimation and the resulted wheel circumferences can be found in Figure 6. The initial parameter values are the nominals $\hat{\vartheta}_{\text {circ }, 0}=\left[c_{e, R L, n o m}, c_{e, R R, \text { nom }}\right]^{T}$. The estimated values are in a $6 \mathrm{~mm}$ range for each wheel and the standard deviation is only $1.4 \mathrm{~mm}$. Therefore the convergence to the true value is occured. The mean position

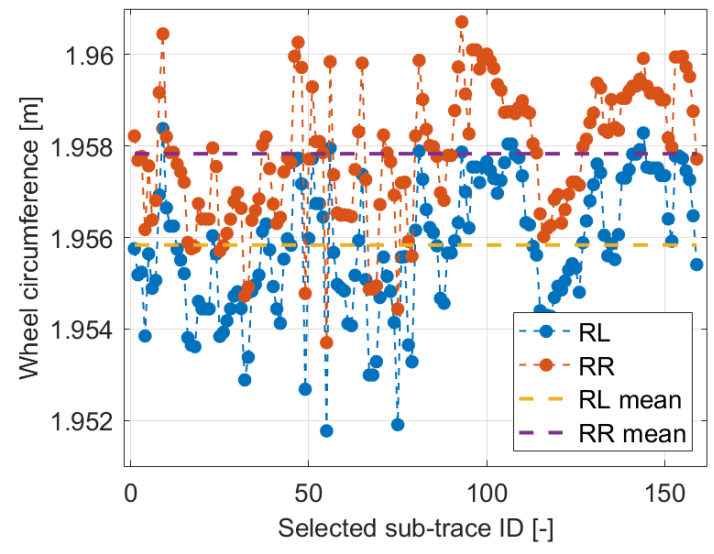

Fig. 6. Estimated circumferences of the initial circumference estimation

errors can be found in Figure 7, with the estimated circumferences the model results 3.5 times lower error, the mean error of the sub-traces is $1.24 \mathrm{~m}$. The difference between the circumferences is $1.99 \mathrm{~mm}$, but despite its low value,

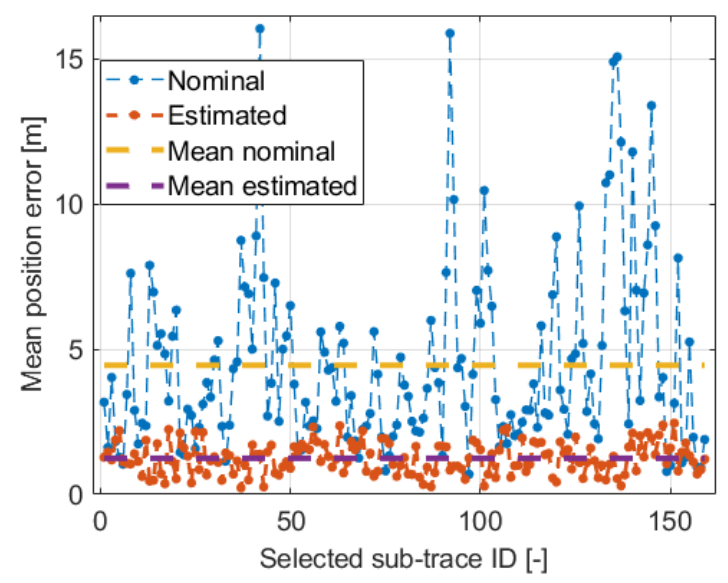

Fig. 7. Position errors of the initial circumference estimation

its impact is significant. The stable estimated value of the circumferences are the mean of the estimated sub-trace ones as $\left[\begin{array}{ll}c_{e, R L, c i r c} & \left.c_{e, R R, c i r c}\right]=\hat{\vartheta}_{\text {circ }, i *}^{t}\end{array}\right.$.

\section{Final estimation of the parameters}

Finally, the track and the load transfer coefficient are identified. In the previous sub-section we can see that the estimated slight circumference difference has a high impact on the position error. However these wheel parameters are calculated with a-priori track and zero deviation ratio parameters, thus these can be local optimums. Therefore with the $t_{R}$ and $D$ parameter the circumferences are also estimated in parallel, with initial value of the previous determined ones. The theta vector contains every parameter $\vartheta_{f i n}=\left[t_{R}, c_{e, R L}, c_{e, R R}, D\right]^{T}$ and its initial value is $\hat{\vartheta}_{\text {fin }, 0}=\left[\begin{array}{llll}t_{R, \text { nom }} & c_{e, R L, \text { circ }} & c_{e, R R, \text { circ }} & D_{\text {nom }}\end{array}\right]$. In Figure 8 the mean position error of the sub-traces of the previous circumference and actual estimation can be found. Only every 4th sub-trace is shown for better view. With the dynamic model the mean error of the sub-traces decreases significantlyto $0.92 \mathrm{~m}$. In Figure 9 the estimated circumfer-

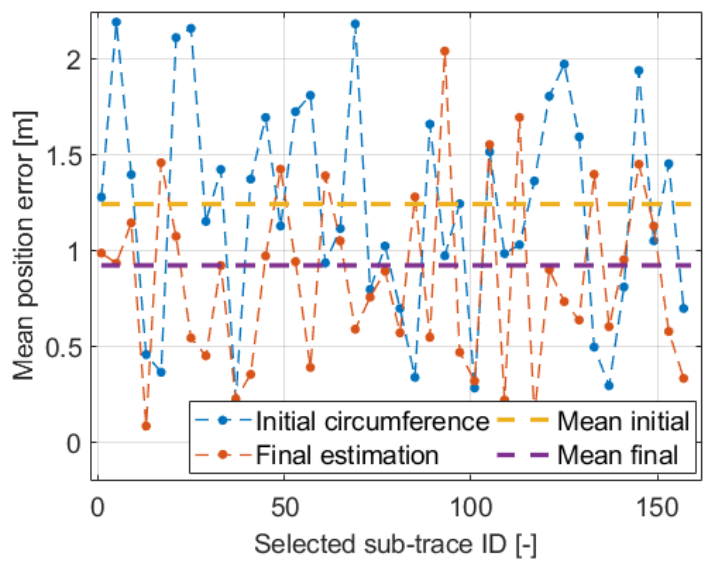

Fig. 8. Position errors the final estimation

ence load transfer coefficient can be found. The mean of the sub-traces is $7.4357 \times 10^{-4} s^{2}$. The standard deviation 
is significant, $1.5845 \times 10^{-4} s^{2}$ which may be related to the previous mentioned extreme situations, therefore further improvement is possible. However, the $0.92 \mathrm{~m}$ mean position

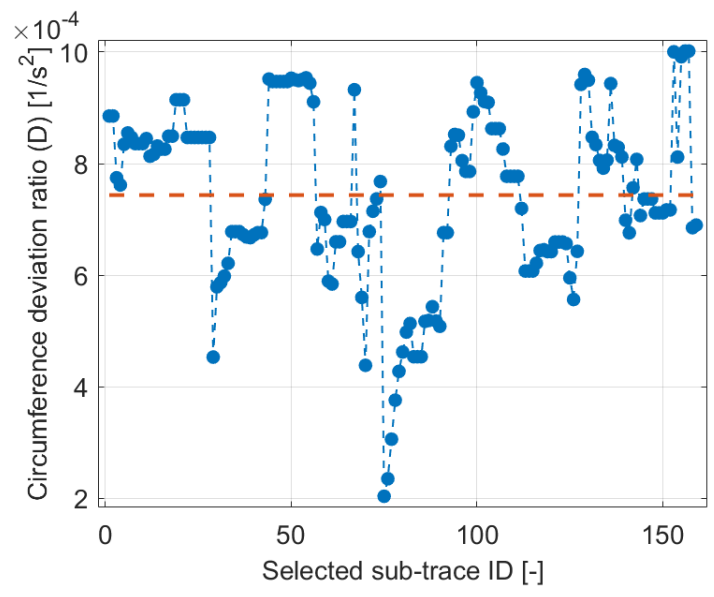

Fig. 9. Estimated load transfer coefficient of the sub-traces

error in a $200 \mathrm{~m}$ long route only with the usage of wheel rotation encoders is an outstanding result.

\section{Final results and test}

The test track is segmented into sub-traces, thus the previous results connecting to the sub-traces, where the used model contains the local estimated parameters. However, this process helps to eliminate the sub-traces containing incorrect measurements or non-modeled errors e.g. wheel slips. But, concrete parameter values are required for the calibrated model, which are the avergare of the sub-trace identified values as

$$
\begin{aligned}
c_{e, R L, o p t} & =1.9558 \mathrm{~m}, \\
c_{e, R R, o p t} & =1.9578 \mathrm{~m}, \\
t_{R, o p t} & =1.5138 \mathrm{~m}, \\
D_{o p t} & =7.4357 \times 10^{-4} \mathrm{~s}^{2} .
\end{aligned}
$$

After the identification the calibrated two-wheel model with the identified parameters is tested on the whole route with different integration time length. With $10 \mathrm{~s}$ the mean route length is $100 \mathrm{~m}$ and the resulted position errors of the nominal and calibrated settings can be found in Figure 10. The mean error with the identified model is $0.67 \mathrm{~m}$, the relative error is $0.58 \%$ and it is 4 times better than the result of the nominal setting.

\section{Conclusions}

The paper proposes an iterative parameter identification algorithm for two-wheel vehicle model taking into account dynamic load transfer. The algorithm results in high accuracy estimation, because the measurement noises have lower effect due to the constructed LS task. The rear circumferences, track width and the load transfer coefficient identified from real measurements of onboard sensors. The main contribution is the improvement of pure kinematic model to account the dynamic effects and the calibration of it from highly

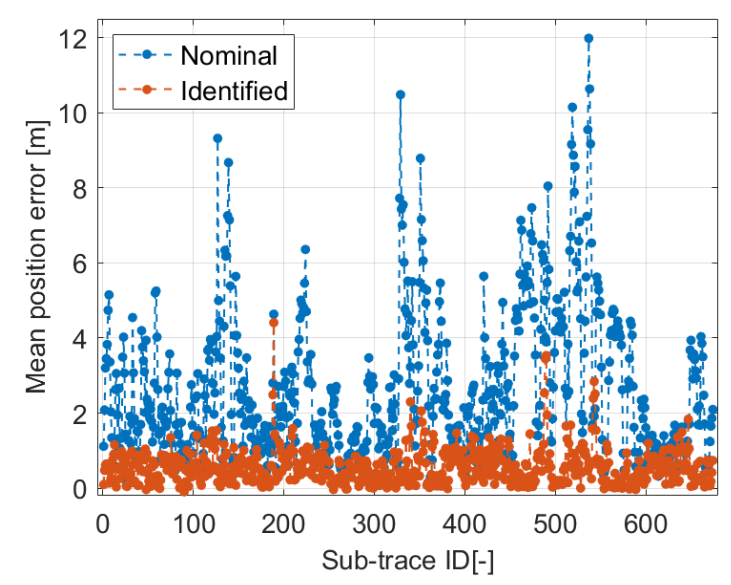

Fig. 10. Position errors of the test

uncertain initial parameter values and noisy measurements. As a future challenge, the applied vehicle odometry model might be improved through the consideration of the side slip.

\section{REFERENCES}

[1] Bloesch, M., Omari, S., and Hutter, M. "Robust visual inertial odometry using a direct EKF-based approach" In IEEE/RSJ International Conference on Intelligent Robots and Systems (IROS), 298-304. 2015

[2] Schanz, A., Spieker, A., and Kuhnert, K.D. "Autonomous parking in subterranean garages a look at the position estimation" In IEEE Intelligent Vehicles Symposium, 253-258. 2003

[3] Kochem, M., Neddenriep, R., Isermann, R., Wagner, N., and Hamann, "Accurate local vehicle dead-reckoning for a parking assistance system" In American Control Conference, 4297-4302. 2002

[4] Brunker, A., Wohlgemuth, T., Frey, M., and Gauterin, F. "Odometry 2.0: A slip-adaptive UIF-based four-wheel-odometry model for parking" In IEEE Transactions on Intelligent Transportation systems. 2017

[5] Fazekas, M., Nemeth, B., and Gaspar, P. "Model based vehicle localization via an iterative parameter estimation" In 26th IAVSD Symposium on Dynamics of Vehicles on Roads and Tracks, 1-10. 2019

[6] Kochem, M., Neddenriep, R., Isermann, R., Wagner, N., and Hamann, C.D. "The clapper: a dual-drive mobile robot with internal correction of dead-reckoning errors" In IEEE International Conference on Robotics and Automation" 3085-3090. 1994

[7] Borenstein, J. "Experimental results from internal odometry error correction with the omnimate mobile robot" In TRANSACTIONS ON ROBOTICS AND AUTOMATION, 14(6), 963-969. 1998

[8] Lee, K. and Chung, W. "Calibration of kinematic parameters of a car-like mobile robot to improve odometry accuracy" In IEEE International Conference on Robotics and Automation 2008

[9] Larsen, T.D., Bak, M., Andersen, N.A., and Ravn, O. "Location estimation for an autonomously guided vehicle using an augmented Kalman-flter to autocalibrate the odometry" In First International Conference on Multisource-Multisensor Information Fusion 1998

[10] Martinelli, A. et al. "Estimating the odometry error of a mobile robot during navigation" In European Conference on Mobile Robots 2003

[11] Caltabiano, D., Muscato, G., and Russo, F. "Localization and selfcalibration of a robot for volcano exploration" In IEEE International Conference on Robotics and Automation, 586-591. 2004

[12] Brunker, A., Wohlgemuth, T., Michael, and Gauterin, F. "GNSSshortages-resistant and self-adaptive rear axle kinematic parameter estimator (SA-RAKPE)" In IEEE Int. Vehicles Symposium, 2017

[13] Crassidis, J.L. and Junkins, J.L. "Optimal Estimation of Dynamic Systems" CRC Press. 2012

[14] Caron, F., Du os, E., Pomorski, D., and Vanheeghe, P. "'GPS/IMU data fusion using multisensor Kalman-filtering: Introduction of contextual aspects" In Information Fusion, 7, 221-230. 2006

[15] Ma, J., Ding, F., Xiong, W., and Yang, E. "Combined state and parameter estimation for hammerstein systems with time delay using the kalman-filtering" In International Journal of Adaptive Control and Signal Processing, 31. 2017 